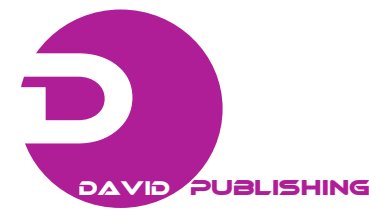

\title{
A Study on the Levitation Control of the Multi-degrees-of-Freedom Rotational Machine Supported by Magnetic Bearings Using Flux Feedback
}

\author{
Jun-Ho Lee \\ Advanced Traction and Noncontact Feeding System Research Team, Korea Railroad Research Institute, Uiwang-City, Gyeonggi-do \\ 437-757, Korea
}

Received: April 01, 2014 / Accepted: April 18, 2014 / Published: June 25, 2014.

\begin{abstract}
This paper deals with an open-loop characteristic of a magnetically levitated system including flux feedback. In order to design a controller to obtain a good disturbance rejection and to be insensitive to parameter variations, it might be useful to employ a flux feedback loop. The air gap flux which can be sensed by a proper sensor has linear relationship with respect to the change of the current and the air gap. This linear property decreases the inherent nonlinearity of the magnetic suspension system that is caused by the coupling between the electrical actuator and the mechanical plant. Simulation results achieved from a multi-degree-of-freedom numerical model show that the flux feedback loop makes an improvement of the performance of the magnetic suspension system against the load variations.
\end{abstract}

Key words: Flux feedback, magnetic bearings, levitation, rotational machine, multi-degrees-of-freedom.

\section{Introduction}

In recent trends to develop various technologies, the word "contactless" has been begun to receive notable attentions because of its effectiveness and maintenance free properties that are impossible to achieve in the conventional "contact" system. The typical representative contactless system is a magnetic suspension system which is commonly known as maglev (magnetically levitated) system. The first trial for the maglev has been performed at the University of Virginia in 1937 which has been intended to test magnetic bearing systems.

Recently, there are various applications employing the magnetic levitation configuration as a core technology, such as the magnetically levitated train system, the high speed turbo compressors, the flywheel energy storage system and the artificial heart pump.

Corresponding author: Jun-Ho Lee, Ph.D., research fields: design and control of flywheel energy storage system, design of wireless power transfer system. E-mail: jhlee77@krri.re.kr.
The magnetically levitated system can be divided into two parts based on the levitation method: one is a repulsive type using super conductors. One of disadvantages of this type of suspension system is that it needs to operate below the critical speed when the suspended object is stationary because an active suspension actuator and a controller are not included which make it possible to pass through the critical speeds; the other type is EMS (electromagnetic suspension system) using ferromagnetic or permanent magnet. The EMS type has one significant advantage in that it provides attraction force at zero speed, but such system is inherently unstable. In order to overcome the inherent instability, an active controller plays a very important role in the electromagnet type suspension system to make the stable suspension and maintain the suspended object within the nominal air gap.

Especially, when external disturbance or parameter variations affect on the active controller of the Maglev, 
the system may cause a malfunction of the suspension system. In such case, a controller that has a robustness property should be introduced, or a system model should be considered to avoid the effects of the external disturbance or parameter variations [1-4].

In many papers and literatures, the design methodologies of the active magnetic suspension controllers have already been presented. Some advances in improving AMB (active magnetic bearing) feedback control using a nonlinear gap model instead of a linear gap model were proven in a SISO (single-output) balance beam test rig [5]. Hu et al. [6] developed a reduced power loss AMB in a balance beam by considering the constraint of voltage saturation as obtained for the class of sinusoidal force signals. Lindlau et al. [7] considered a feedback linearization voltage mode controller in an AMB balance beam using mu synthesis. Lin et al. [8] developed a model which treated coil resistance changes with structured uncertainty as well as considering actuator flux leakage. Nonlinear output regulation for a balance beam was carried out in a similar balance beam. Issues with a high speed energy storage flywheel AMB concerning modeling uncertainty and treatment using mu synthesis control were reported in Ref. [9].

However, the papers for the analysis of the open-loop characteristics of the magnetic suspension system have rarely presented. A significant paper on flux feedback control of AMBs used current control with both displacement and the deviation flux expressed as a linear combination of the total force in the AMB $[10,11]$. At the equilibrium point, the total force in the AMB should be equal to the sum of the state feedback control effort and the estimated disturbance force. A linear flux observer was developed to reject a static and periodic disturbance force [10].

Sometimes, it is very valuable to make focus on the inherent properties of the magnetic suspension system to achieve a design of the improved active controller against the system parameter variations. The purpose of this paper is to show the effectiveness of the flux feedback loop of the air gap in the open loop characteristic of the magnetic suspension system when a constant voltage is applied to the electromagnet coils.

The paper is organized as follows: Section 2 summarizes the fundamental mathematical model (one-degree-of-freedom and four-degrees-of-freedom) of the EMS system, which does not include the flux feedback loop; Section 3 shows a modified mathematical model including the flux feedback loop. In the flux feedback loop, the estimated flux is linearly proportional to the magnetic excitation current and inversely proportional to the air gap; Section 4 presents that the magnetic suspension system including flux a loop has robustness against the parameter variations based on the simulation results; and Section 5 gives conclusions.

\section{Fundamental Mathematical Model}

\subsection{One-Degree-of-Freedom}

Fig. 1 shows a simple schematic diagram for EMS system which has the electromagnets as the suspension actuators.

The mathematical model of this system is divided into two parts: one is the plant (mechanical) dynamics and the other is the actuator dynamics. The plant

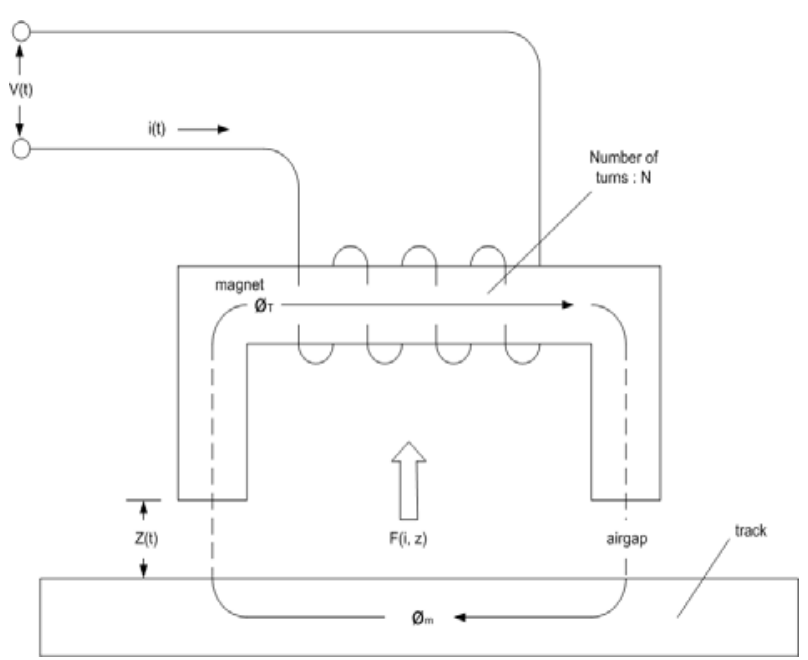

Fig. 1 Schematic diagram for EMS system. 
(mechanical) dynamics is

$$
m \ddot{z}=F(i, z)-m g-f_{d}
$$

where, $m$ is the total mass of the controlled object, $g$ is the gravitational acceleration and $f_{d}$ is the external disturbance force acting on the controlled object. In Eq. (1), $F(i, z)$ is the electromagnets attraction force which is proportional to the current deviation and inversely proportional to the air gap deviation, expressed such as:

$$
F(i, z)=\frac{B^{2} A}{\mu_{0}}=\frac{\mu_{0} N^{2} A}{4}\left(\frac{i(t)}{z(t)}\right)^{2}
$$

where, $B$ is the flux density of the magnetic core material, $A$ is the cross sectional area of the pole face of the electromagnet, $\mu_{0}$ is the permeability in the air space and $N$ is the number of turns. In order to drive Eq. (2), it is necessary to check the relation between the inductance and the magnetic flux density.

$$
L(i, z)=\frac{N}{i} \varnothing_{m}=\frac{N}{i(t)} \frac{N i(t)}{R_{T}}
$$

where, $R_{t}=\frac{2 z(t)}{\mu_{0} A}=\frac{V_{T}}{\emptyset_{m}}$ is the reluctance of the magnetic circuit and $V_{T}$ is the electromotive force. Normally the reluctance in the magnetic core is assumed to be negligible compared with the air gap, thus the coil inductance becomes

$$
L(i, z)=\frac{\mu_{0} N^{2} A}{2 z(t)}
$$

$L i(t)=$ NBA and Eq. (4) yield the magnetic flux density such as:

$$
B=\frac{L i(t)}{N A}=\frac{N^{2} \mu_{0} A i(t)}{2 z(t) N A}=\frac{\mu_{0} N i(t)}{2 z(t)}
$$

Substituting Eq. (5) into $F(i, z)=\frac{B^{2} A}{\mu_{0}}$ yields Eq. (2). Eq. (2) has high nonlinearity and it is not easy to use Eq. (2) without doing the linear approximation with respect to the nominal point $\left(i_{0}, z_{0}\right)$. For the linear approximation, the Taylor series expansion is usually employed. From the Taylor series expansion, Eq. (2) becomes

$$
F(i, z)=k_{i} i(t)-k_{z} z(t)
$$

where, $\quad k_{z}=\frac{\mu_{0} N^{2} A i_{0}^{2}}{2 z_{0}^{3}}$ and $\quad k_{i}=\frac{\mu_{0} N^{2} A i_{0}}{2 z_{0}^{2}}$ are the coefficients for the linear approximation of Eq. (2). $k_{z}$ and $k_{i}$ represent the displacement stiffness and the current stiffness, respectively.

In Eq. (6), the stiffness $k_{z}$ has negative sign which means that once the attractive force of the electromagnets is activated, the controlled object is attracted until the electromagnets stop attracting the controlled object. This is one of the reasons why the electromagnet suspension system should have the active controller to control the air gap deviation.

The actuator dynamics is

$$
\begin{aligned}
V(t) & =R i(t)+\frac{d}{d t}[L(i, z) i(t)] \\
& =R i(t)+\frac{\mu_{0} N^{2} A}{2 z(t)} \frac{d}{d t} i(t)-\frac{\mu_{0} N^{2} A i_{0}}{2 z(t)^{2}} \frac{d}{d t} z(t)
\end{aligned}
$$

where, $v$ is the coil voltage, $R$ is the coil resistance, and $L(i, z)$ is the coil inductance which is the function of the air gap displacement. It should be noted that there is a variation of the inductance with respect to the air gap displacement in the second term, and that the third term denotes a voltage which varies with changes in the air gap $z(t)$ and its rate of change similar to back EMF voltage. By using Eqs. (1), (6) and (7), we can drive a state space equation such as:

$$
\left[\begin{array}{c}
\dot{z} \\
\ddot{z} \\
i
\end{array}\right]=\left[\begin{array}{ccc}
0 & 1 & 0 \\
\frac{-k_{z}}{m} & 0 & \frac{k_{i}}{m} \\
0 & \frac{k_{z}}{k_{i}} & \frac{-R}{L}
\end{array}\right]\left[\begin{array}{c}
z \\
\dot{z} \\
i
\end{array}\right]+\left[\begin{array}{c}
0 \\
0 \\
\frac{1}{L}
\end{array}\right] V+\left[\begin{array}{c}
0 \\
\frac{1}{m} \\
0
\end{array}\right] f_{d}(8)
$$

Fig. 2 shows the simple block diagram for the open-loop EMS system which does not include the flux feedback signal. The transfer function of the open loop system is induced by the Laplace transform of Eq. (8) for each state variables, which is

$$
z(s)=\left[\frac{\frac{k_{i}}{m R}}{\left(1+\frac{L}{R} s\right)\left(s^{2}+\frac{k_{i}}{m R\left(1+\frac{L}{R} s\right)} s-\frac{k_{z}}{m}\right)}\right] V(s)
$$




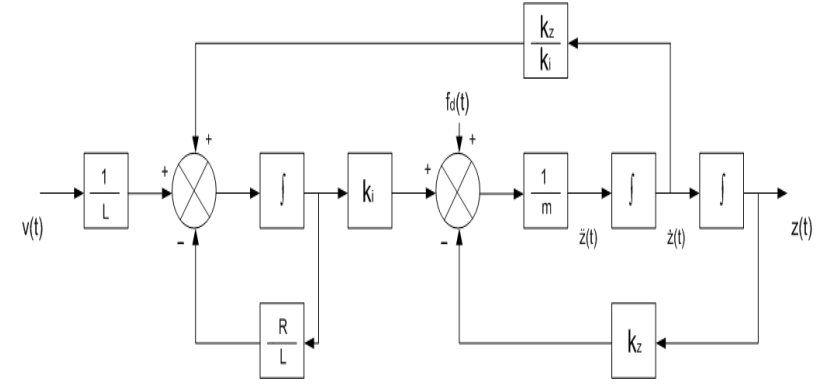

Fig. 2 Open-loop diagram without flux.

\subsection{Four-Degrees-of-Freedom}

Fig. 3 presents a rotational machine supported by the magnetic bearings which has four-degrees-of-freedom. For the rotational rotor, left and right electromagnetic bearings support the rotor to make levitation so that the rotor can rotate without any contact with the stator. Displacement sensors are very close attached to the electromagnetic bearings to make feedback of the displacement deviation which make it possible for the rotor to be controller actively. Equation of motion of the rotor shown in Fig. 3 is such as:

$$
\begin{gathered}
M \ddot{q}+G \dot{q}={ }_{M} T_{B} u_{A M B} \\
y={ }_{S} T_{M} q \\
M=\left[\begin{array}{cccc}
I_{y} & 0 & 0 & 0 \\
0 & m & 0 & 0 \\
0 & 0 & I_{x} & 0 \\
0 & 0 & 0 & m
\end{array}\right] G=\left[\begin{array}{cccc}
0 & 0 & I_{z} \Omega & 0 \\
0 & 0 & 0 & 0 \\
-I_{z} \Omega & 0 & 0 & 0 \\
0 & 0 & 0 & 0
\end{array}\right](11) \\
T_{B}=\left[\begin{array}{llll}
a & b & 0 & 0 \\
1 & 1 & 0 & 0 \\
0 & 0 & a & b \\
0 & 0 & 1 & 1
\end{array}\right]{ }_{S} T_{M}=\left[\begin{array}{llll}
c & 1 & 0 & 0 \\
d & 1 & 0 & 0 \\
0 & 0 & c & 1 \\
0 & 0 & d & 1
\end{array}\right] \\
q=(\beta, x,-\alpha, y)^{T} \\
u_{A M B}=\left(f_{x A}, f_{x B}, f_{y A}, f_{y B}\right)^{T} \\
y=\left(x x_{s A}, x_{s B}, y_{s A}, y_{s B}\right)^{T}
\end{gathered}
$$

where, $M$ is the mass matrix that is composed of mass and moment of inertia, $G$ is the gyroscope matrix that is 0 when the rotor stops, however the amplitude gradually becomes greater as the rotational speed goes up. ${ }_{M} T_{B}$ is the transformation matrix to transform the bearing coordinates to mass center coordinates, and

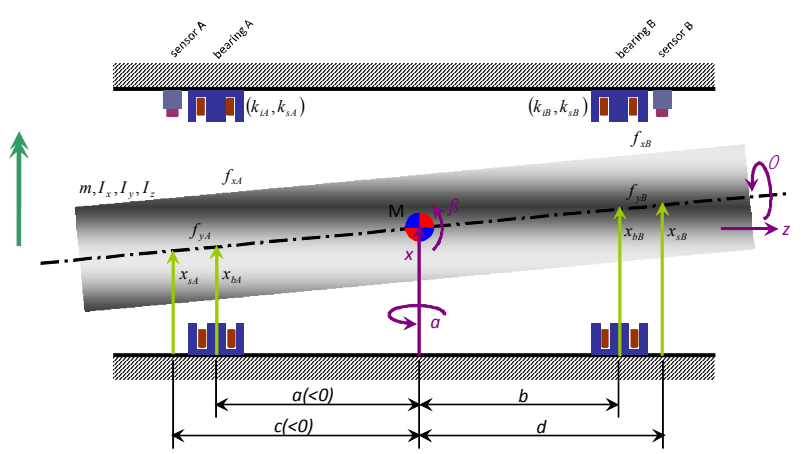

Fig. 3 Conceptual diagram of the rotational machine which has four-degree-of-freedom.

${ }_{S} T_{M}$ is the transformation matrix to transform mass center coordinates to displacement sensor coordinates. $q$ is the vector that expresses the position of the radial directions and the rotational angles in the mass center coordinates. $u_{A M B}$ is the force that is generated from the magnetic bearings, and $y$ is the distance that is measured from the displacement sensor to the rotor.

$u_{A M B}$ should be linearized as shown in the fundamental formulas of the one-degree-of-freedom model. Eq. (13) represents the linearized model of the four-degrees-of-freedom model. In Eq. (13), $K_{s}$ is the displacement stiffness that makes the system unstable as presented in Eq. (6), $K_{i}$ is the current stiffness, $q_{B}$ is the vector that indicates the distance from the bearing location to the rotor, and $i$ is the current vector for the bearings.

$$
\begin{aligned}
u_{A M B} & =\left[\begin{array}{l}
f_{x A} \\
f_{x B} \\
f_{y A} \\
f_{y B}
\end{array}\right]=-\left[\begin{array}{cccc}
k_{s A} & 0 & 0 & 0 \\
0 & k_{s B} & 0 & 0 \\
0 & 0 & k_{s A} & 0 \\
0 & 0 & 0 & k_{s B}
\end{array}\right]\left[\begin{array}{c}
x_{b A} \\
x_{b B} \\
y_{b A} \\
y_{b B}
\end{array}\right] \\
& +\left[\begin{array}{cccc}
k_{i A} & 0 & 0 & 0 \\
0 & k_{i B} & 0 & 0 \\
0 & 0 & k_{i A} & 0 \\
0 & 0 & 0 & k_{i B}
\end{array}\right]\left[\begin{array}{c}
i_{x A} \\
i_{x B} \\
i_{y A} \\
i_{y B}
\end{array}\right] \\
& =-K_{s} q_{B}+K_{i} i
\end{aligned}
$$

From Eqs. (10) and (13), the linearized equation of motion is such as:

$$
M \ddot{q}+G \dot{q}={ }_{M} T_{B}\left(-K_{s} q_{B}+K_{i} i\right)
$$

The vector $q_{B}$ is transformed to mass center coordinates by using the transformation matrix ${ }_{M} T_{B}$ 
which is expressed as:

$$
\begin{aligned}
q_{B} & =\left[\begin{array}{c}
x_{b A} \\
x_{b B} \\
y_{b A} \\
y_{b B}
\end{array}\right]=\left[\begin{array}{llll}
a & 1 & 0 & 0 \\
b & 1 & 0 & 0 \\
0 & 0 & a & 1 \\
0 & 0 & b & 1
\end{array}\right]\left[\begin{array}{c}
\beta \\
x \\
-\alpha \\
y
\end{array}\right] \\
& ={ }_{B} T_{M} q=\left({ }_{M} T_{B}\right)^{T} q
\end{aligned}
$$

Substituting Eq. (15) for Eq. (14) yields:

$$
\begin{aligned}
& M \ddot{q}+G \dot{q}=-{ }_{M} T_{B} K_{S} T_{B}^{T} q+{ }_{M} T_{B} K_{i} i=-K_{S M} q+{ }_{M} T_{B} K_{i} i(16) \\
& M \ddot{q}+G \dot{q}+K_{S M} q={ }_{M} T_{B} K_{i} i
\end{aligned}
$$

where,

$$
\begin{aligned}
& K_{S M}={ }_{M} T_{B} K_{S M} T_{B}^{T} \\
& =\left[\begin{array}{cccc}
k_{S A} a^{2}+k_{S B} b^{2} & k_{S A} a+k_{S B} b & 0 & 0 \\
k_{S A} a+k_{S B} b & k_{S A}+k_{S B} & 0 & 0 \\
0 & 0 & k_{S A} a^{2}+k_{S B} b^{2} & k_{S A} a+k_{S B} b \\
0 & 0 & k_{S A} a+k_{S B} b & k_{S A}+k_{S B}
\end{array}\right]
\end{aligned}
$$

As seen in Eq. (16), the mathematical model of the four-degrees-of-freedom rotational machine supported by magnetic bearings is very similar to the 2 nd order mechanical system. However, the rotor shaft is coupled due to the gyroscopic effect and the non-colocation problem between the displacement sensors and the magnetic bearing actuators as shown in Eq. (16).

Voltage equations without flux feedback are expressed in Eq. (17).

$$
V=R i+L \frac{d i}{d t}-K_{i} \frac{d q_{B}}{d t}
$$

Eq. (17) can be modified to present matrix pattern as:

$$
\begin{gathered}
{\left[\begin{array}{c}
V_{x A} \\
V_{x B} \\
V_{y A} \\
V_{y B}
\end{array}\right]=\left[\begin{array}{cccc}
R_{A} & 0 & 0 & 0 \\
0 & R_{B} & 0 & 0 \\
0 & 0 & R_{A} & 0 \\
0 & 0 & 0 & R_{B}
\end{array}\right]\left[\begin{array}{c}
i_{x A} \\
i_{x B} \\
i_{y A} \\
i_{y B}
\end{array}\right]} \\
+\left[\begin{array}{cccc}
L_{A} & 0 & 0 & 0 \\
0 & L_{B} & 0 & 0 \\
0 & 0 & L_{A} & 0 \\
0 & 0 & 0 & L_{B}
\end{array}\right]\left[\begin{array}{c}
\dot{i}_{x A} \\
i_{x B} \\
i_{y A} \\
i_{y B}
\end{array}\right] \\
-\left[\begin{array}{cccc}
k_{i A} & 0 & 0 & 0 \\
0 & k_{i B} & 0 & 0 \\
0 & 0 & k_{i A} & 0 \\
0 & 0 & 0 & k_{i B}
\end{array}\right]\left[\begin{array}{l}
x_{b A} \\
x_{b B} \\
y_{b A} \\
y_{b B}
\end{array}\right]
\end{gathered}
$$

From Eq. (17) and by using transformation matrix ${ }_{M} T_{B}$, current slew rate is derived.

$$
\begin{aligned}
\frac{\mathrm{d} i}{\mathrm{~d} t} & =L^{-1} V-L^{-1} R i+K_{i}^{-1} K_{s} \frac{\mathrm{d} q_{B}}{\mathrm{~d} t} \\
& =L^{-1} V-L^{-1} R i+K_{i}^{-1} K_{s}{ }_{M} T_{B}^{T} \frac{\mathrm{d} q}{\mathrm{~d} t}
\end{aligned}
$$

The state space model for the four-degrees-of-freedom rotational machine can be presented by using Eqs. (17)-(19).

$$
\begin{aligned}
{\left[\begin{array}{c}
\dot{q} \\
\dot{q} \\
i
\end{array}\right] } & =\left[\begin{array}{ccc}
0 & I & 0 \\
-M^{-1} K_{s M} & -M^{-1} G & -M^{-1}{ }_{M} T_{B} K_{i} \\
0 & K_{i}^{-1} K_{s} T_{B}^{T} & -L^{-1} R
\end{array}\right]\left[\begin{array}{c}
q \\
\dot{q} \\
i
\end{array}\right](20) \\
& +\left[\begin{array}{c}
0 \\
0 \\
L^{-1} V
\end{array}\right]
\end{aligned}
$$

\section{Flux Feedback Loop}

\subsection{One-Degree-of-Freedom}

In this section, estimation of the open loop characteristics using flux feedback is presented. The air gap flux which is produced by the core magnet is proportional to the pole face area as:

$$
\begin{aligned}
\Phi & =B A \\
& =\frac{\mu_{0} N A i}{2 z}
\end{aligned}
$$

The time derivative of $\Phi$ yields

$$
\frac{\mathrm{d}}{\mathrm{d} t} \Phi=\frac{\mu_{0} A N}{2 z} \frac{\mathrm{d} i}{\mathrm{~d} t}+\frac{\mu_{0} A N i_{0}}{2 z^{2}} \frac{\mathrm{d} z}{\mathrm{~d} t}
$$

If the magnetic flux $\Phi$ is fed back to the amplifier that activates the electromagnets the terms for the dynamics of the electromagnet actuators should be modified so that the force component which is included in the magnetic flux $\Phi$ should be included in the actuator dynamic equation. This yields Eq. (23).

$$
\begin{aligned}
v(t)= & R i(t)+\frac{\mathrm{d}}{\mathrm{d} t}[L(i, z) i(t)]+k_{\Phi \Phi} N \Delta \Phi_{a} \\
= & R i(t)+\frac{\mu_{0} N^{2} A}{2 z(t)} \frac{\mathrm{d}}{\mathrm{d} t} i(t)-\frac{\mu_{0} N^{2} A i_{0}}{2 z(t)^{2}} \frac{\mathrm{d}}{\mathrm{d} t} z(t) \\
& +k_{\Phi \Phi}\left(k_{f i} i(t)-k_{f z} z(t)\right) \\
= & R i(t)+L i(t)-k_{i} \dot{z}(t)+k_{\Phi \Phi}\left(k_{f i} i(t)-k_{f z} z(t)\right)
\end{aligned}
$$


where, $k_{f i}=\frac{\mu_{0} A N^{2}}{2 z_{0}}, \quad k_{f z}=\frac{\mu_{0} A N^{2} i_{0}}{2 z_{0}^{2}}$ and $k_{\Phi \Phi}$ is flux feedback gain.

Compare to Eq. (7), the flux feedback term is included in Eq. (23) which is expressed as linear combination of the coil current and air gap deviation. A combination of Eqs. (1), (6) and (12) induces the state space model including the flux feedback such as:

$$
\begin{aligned}
{\left[\begin{array}{c}
\dot{z} \\
\ddot{z} \\
\dot{i}
\end{array}\right]=\left[\begin{array}{ccc}
0 & 1 & 0 \\
\frac{-k_{z}}{m} & 0 & \frac{k_{i}}{m} \\
\frac{k_{\Phi \Phi} k_{f z}}{L} & \frac{k_{z}}{k_{i}} & \frac{\left(-R-k_{\Phi \Phi} k_{f i}\right)}{L}
\end{array}\right]\left[\begin{array}{c}
z \\
\dot{z} \\
i
\end{array}\right] } \\
+\left[\begin{array}{c}
0 \\
0 \\
\frac{1}{L}
\end{array}\right] v+\left[\begin{array}{c}
0 \\
\frac{1}{m} \\
0
\end{array}\right] f_{d}
\end{aligned}
$$

Fig. 4 presents the open-loop diagram with flux feedback loop. In Fig.4, $k_{\Phi \Phi}$ represents the flux feedback gain.

From the characteristic equation of Eq. (24), the gain of the flux feedback loop $k_{\Phi \Phi}$ can be adjusted such that

$$
k_{\Phi \Phi}=-\frac{k_{z} R}{k_{i} k_{z i}-k_{z} k_{i z}}
$$

The input-output transfer function is reduced to

$$
z(s)=\left[\frac{\frac{k_{i}}{m} L}{s^{2}\left\{s+\frac{R}{L}+\frac{k_{\Phi \Phi} k_{f i}}{L}\right\}}\right] v(s)
$$

which indicates that flux feedback makes the system conditionally stable due to free from the $k_{z}$ parameter variation.

\subsection{Four-Degrees-of-Freedom}

Voltage equations with flux feedback are expressed in Eq. (27) which is the same as Eq. (23) except for the number of degrees of freedom.

$$
V=R i+L \frac{d i}{d t}-K_{i} \frac{d q_{B}}{d t}+K_{\varphi \varphi} K_{f i} i-K_{\varphi \varphi} K_{f z} q_{B}
$$

Matrix pattern of Eq. (27) and current slew rate are expressed in Eqs. (28) and (29), respectively.

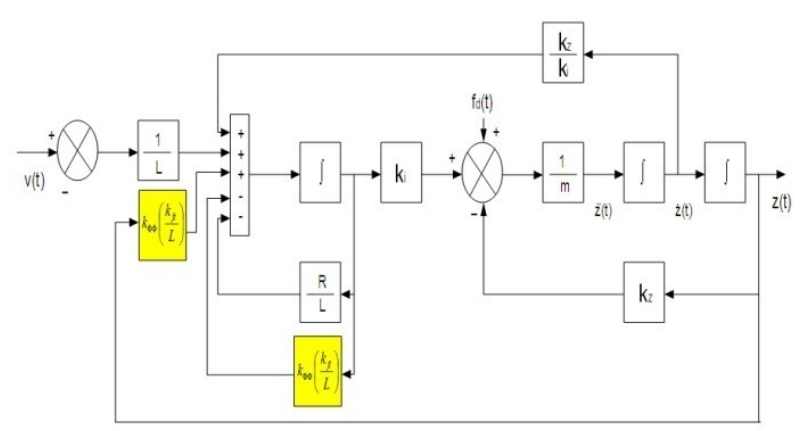

Fig. 4 Open-loop diagram with flux feedback.

$$
\begin{aligned}
& {\left[\begin{array}{c}
V_{x A} \\
V_{x B} \\
V_{y A} \\
V_{y B}
\end{array}\right]=\left[\begin{array}{cccc}
R_{A} & 0 & 0 & 0 \\
0 & R_{B} & 0 & 0 \\
0 & 0 & R_{A} & 0 \\
0 & 0 & 0 & R_{B}
\end{array}\right]\left[\begin{array}{c}
i_{x A} \\
i_{x B} \\
\dot{i}_{y A} \\
i_{y B}
\end{array}\right]+\left[\begin{array}{cccc}
L_{A} & 0 & 0 & 0 \\
0 & L_{B} & 0 & 0 \\
0 & 0 & L_{A} & 0 \\
0 & 0 & 0 & L_{B}
\end{array}\right]\left[\begin{array}{c}
\dot{i}_{x A} \\
i_{x B} \\
i_{y A} \\
i_{y B}
\end{array}\right]} \\
& -\left[\begin{array}{cccc}
k_{i A} & 0 & 0 & 0 \\
0 & k_{i B} & 0 & 0 \\
0 & 0 & k_{i A} & 0 \\
0 & 0 & 0 & k_{i B}
\end{array}\right]\left[\begin{array}{c}
x_{b A} \\
x_{b B} \\
y_{b A} \\
y_{b B}
\end{array}\right]+\left[\begin{array}{cccc}
k_{\phi \phi} k_{f A} & 0 & 0 & 0 \\
0 & k_{\phi \varphi p} k_{f i B} & 0 & 0 \\
0 & 0 & k_{\phi \varphi} k_{f A} & 0 \\
0 & 0 & 0 & k_{\phi \phi p} k_{f B}
\end{array}\right]\left[\begin{array}{c}
i_{x A} \\
i_{x B} \\
i_{y A} \\
i_{y B}
\end{array}\right] \\
& -\left[\begin{array}{cccc}
k_{\phi \varphi} k_{f F A} & 0 & 0 & 0 \\
0 & k_{\phi \varphi} k_{f B B} & 0 & 0 \\
0 & 0 & k_{\phi \varphi} k_{f A A} & 0 \\
0 & 0 & 0 & k_{\phi \varphi} k_{f E B}
\end{array}\right]\left[\begin{array}{c}
x_{b A} \\
x_{b B} \\
y_{b A} \\
y_{b B}
\end{array}\right] \\
& \frac{\mathrm{d} i}{\mathrm{~d} t}=L^{-1} V-L^{-1} R i-K_{i}^{-1} K_{s} \frac{\mathrm{d} q_{B}}{\mathrm{~d} t}-L^{-1} K_{\varphi \varphi} K_{f i} i+L^{-1} K_{\varphi \varphi} K_{f z} q_{B} \\
& =L^{-1} V-L^{-1} R i-K_{i}^{-1} K_{s} M_{B}^{T} \frac{\mathrm{d} q}{\mathrm{~d} t}-L^{-1} K_{\varphi \varphi} K_{f i} i+L^{-1} K_{\varphi \varphi} K_{f z} T_{B}^{T} q
\end{aligned}
$$

where,

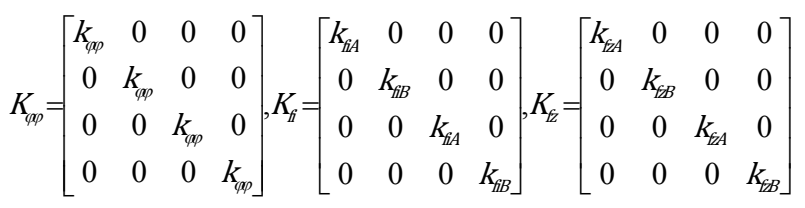

From Eqs. (28) and (29), the state space equations are

$$
\left[\begin{array}{c}
\dot{q} \\
\dot{q} \\
i
\end{array}\right]=\left[\begin{array}{ccc}
0 & I & 0 \\
-M^{-1} K_{s M} & -M^{-1} G & -M^{-1}{ }_{M} T_{B} K_{i} \\
L^{-1} K_{\varphi \phi} K_{f Z L} T_{B}^{T} & -K_{i}^{-1} K_{s M} T_{B}^{T} & -L^{-1} R-L^{-1} K_{\varphi \phi} K_{f i}
\end{array}\right]\left[\begin{array}{c}
q \\
\dot{q} \\
i
\end{array}\right]+\left[\begin{array}{c}
0 \\
0 \\
L^{-1} V
\end{array}\right]
$$

In comparison with Eqs. (20) and (30), Eq. (30) has $k_{\Phi \Phi}, k_{f z}, k_{f i}$ in displacement and current terms which make the system stable, that is similar to Eq. (24).

\section{Simulations}

For the simulations to estimate the flux feedback properties of the electromagnet suspension system, the following parameters are set as in Tables 1 and 2 that 
indicate one-degree-of-freedom system and four-degrees-of-freedom system, respectively. In the simulations to assess the effectiveness of the flux feedback loop, load variations in mass are considered.

Figs. 5 and 6 show the air gap deviation of the one-degree-of-freedom system for the case of when the load acting on the controlled object is changed. When the load is $100 \mathrm{~kg}$ (Fig. 5) without the flux feedback loop, the controlled object contacts (dashed line) the electromagnets at time $50 \mathrm{~s}$. In case of $500 \mathrm{~kg}$ (Fig. 6), it takes more time to contact the electromagnets than that of Fig. 5 due to the heavier load, however, the vibration amplitude becomes bigger and bigger with time. This is because of the inherent unstable characteristics of the magnetic suspension system.

On the contrary, the simulation results with flux feedback loop shown in Figs. 7 and 8 present very good bounded characteristics against the load variation. Fig. 7 is for the case when the load is $100 \mathrm{~kg}$. In Fig. 7, very good robustness property is shown when there is a parameter variation of the EMS system. The small vibration in Fig. 7 is because there

Table 1 Parameters for the one-degree-of-freedom system.

\begin{tabular}{lll}
\hline Variables & Value & Unit \\
\hline Mass: $m$ & 100,500 & $(\mathrm{Kg})$ \\
Coil inductance: $L$ & 72 & $(\mathrm{mH})$ \\
Coil resistance: $R$ & 0.7 & $(\Omega)$ \\
Steady current: $i_{0}$ & 1 & $(\mathrm{~A})$ \\
Cross sectional area: $A$ & $2.2 \times 10^{-6}$ & $\left(\mathrm{~m}^{2}\right)$ \\
Number of turns: $N$ & 140 & $($ turns $)$ \\
Permeability: $\mu_{0}$ & $4 \pi \times 10^{-7}$ & $(\mathrm{H} / \mathrm{m})$ \\
\hline
\end{tabular}

Table 2 Parameters for the four-degrees-of-freedom system.

\begin{tabular}{lll}
\hline Variables & Value & Unit \\
\hline Mass: $m$ & 100,500 & $(\mathrm{Kg})$ \\
Coil inductance: $L$ & 50 & $(\mathrm{mH})$ \\
Coil resistance: $R$ & 1 & $(\Omega)$ \\
Steady current: $i_{0}$ & 1 & $(\mathrm{~A})$ \\
Cross sectional area: $A$ & $1.666 \times 10^{-4}$ & $\left(\mathrm{~m}^{2}\right)$ \\
Number of turns: $N$ & 152 & $($ turns $)$ \\
Permeability: $\mu_{0}$ & $4 \pi \times 10^{-7}$ & $(\mathrm{H} / \mathrm{m})$ \\
\hline
\end{tabular}

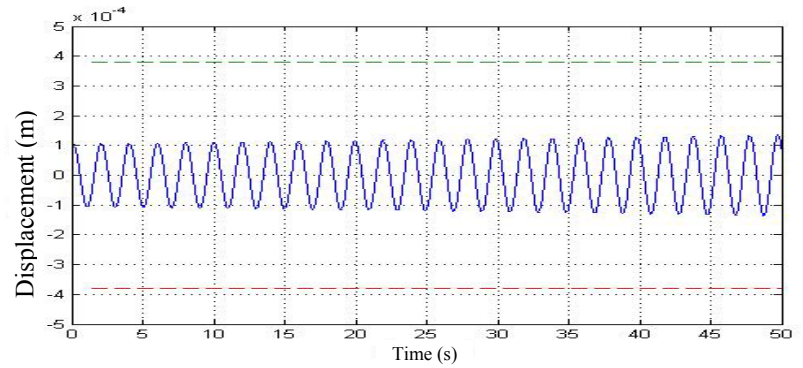

Fig. 5 Gap deviation without flux feedback for one-degree-of-freedom $(m=100 \mathrm{~kg})$.

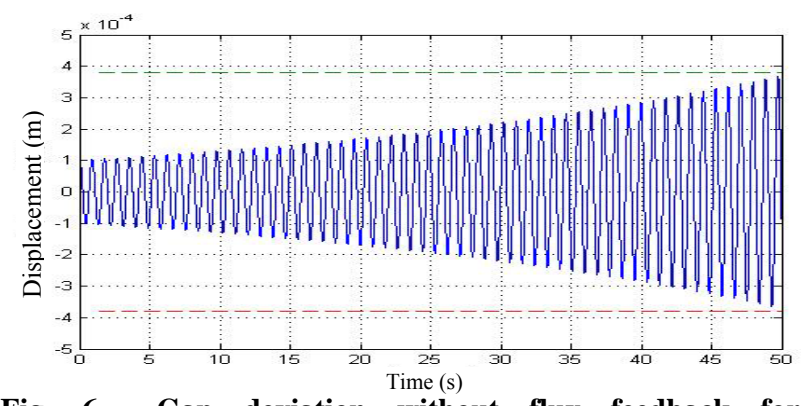

Fig. 6 Gap deviation without flux feedback for one-degree-of-freedom $(m=500 \mathrm{~kg})$.

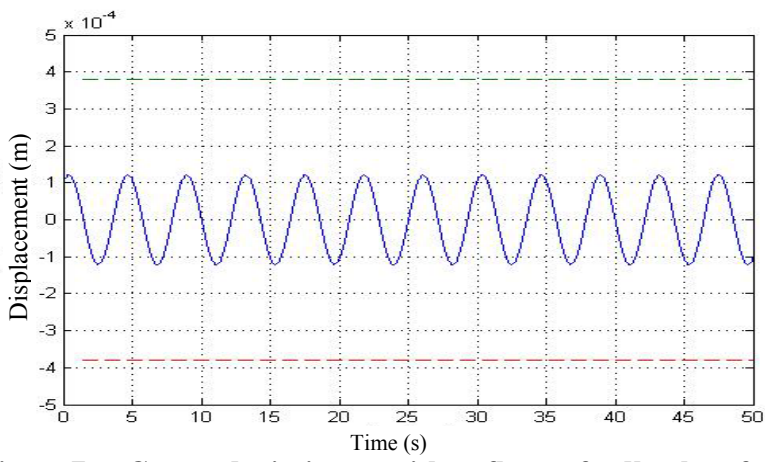

Fig. 7 Gap deviation with flux feedback for one-degree-of-freedom $(m=100 \mathrm{~kg})$.

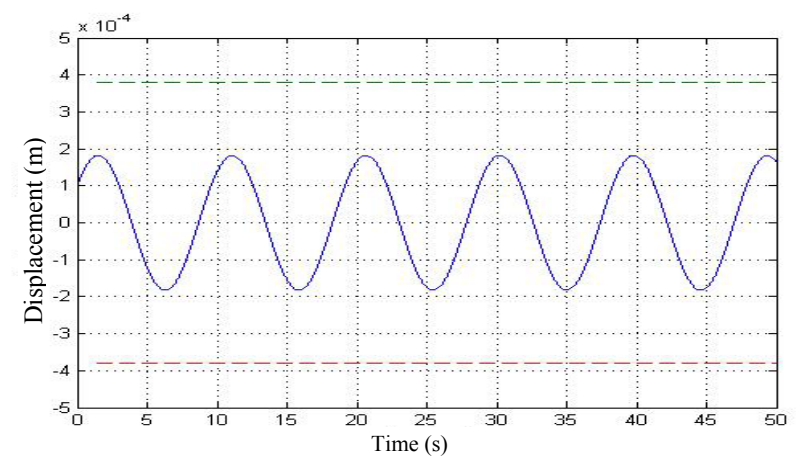

Fig. 8 Gap deviation with flux feedback for one-degree-of-freedom $(m=500 \mathrm{~kg})$.

is no feedback active controller. Thus, any kind of feedback controller can eliminate this vibration. In Fig. 8 , we can see slower vibration and bigger amplitude 

Magnetic Bearings Using Flux Feedback

than that of Fig. 7. This is because of the much heavier load $(500 \mathrm{~kg})$.

Figs. 9-16 are the simulation results for the four-degrees-of-freedom system that includes load variations in mass $(100 \mathrm{~kg}, 500 \mathrm{~kg})$. The gap deviations in the $x$ and $y$ directions that the flux feedback loop is not applied are shown in Figs. 9-12. As seen in Figs. 9-12, due the no flux feedback loop, the system is not bounded, however as shown in Figs.

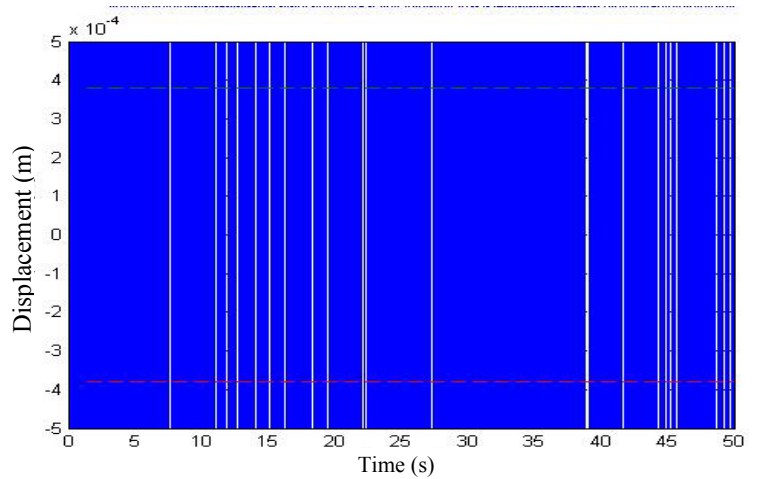

Fig. 9 Gap deviation without flux feedback for four-degrees-of-freedom $(m=100 \mathrm{~kg}, x$ direction).

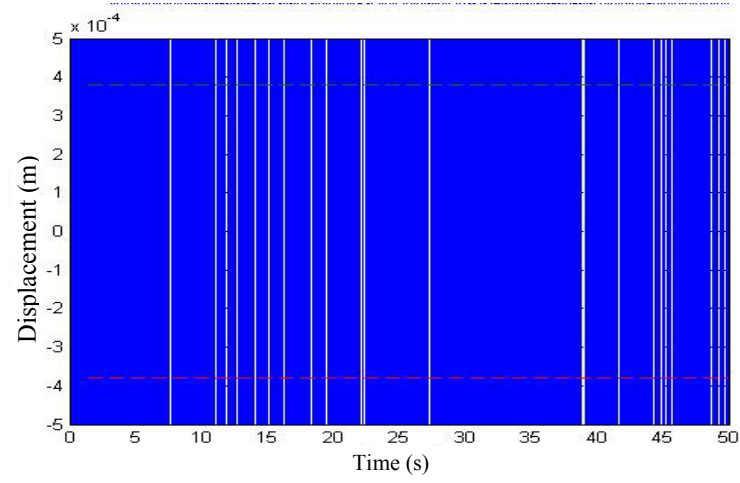

Fig. 10 Gap deviation without flux feedback for four-degrees-of-freedom ( $m=100 \mathrm{~kg}$, $y$ direction).

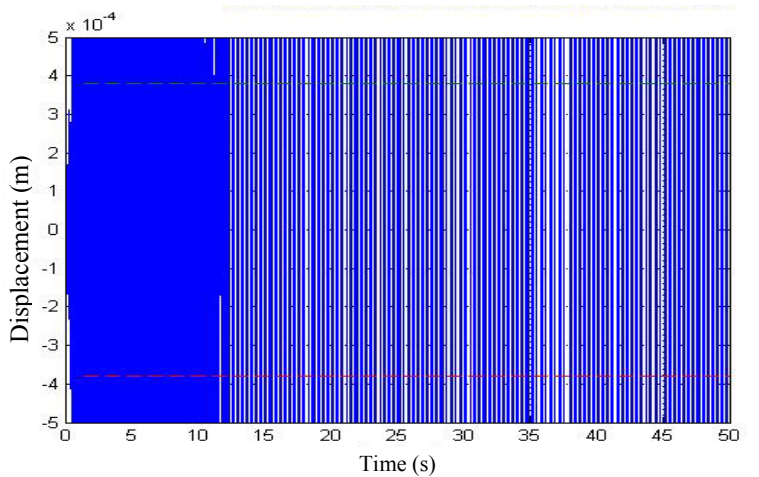

Fig. 11 Gap deviation without flux feedback for four-degrees-of-freedom $(m=500 \mathrm{~kg}, x$ direction).

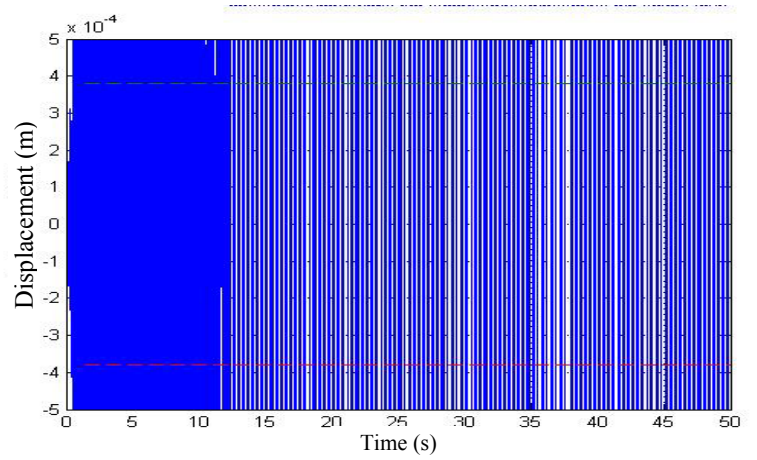

Fig. 12 Gap deviation without flux feedback for four-degrees-of-freedom $(m=500 \mathrm{~kg}, y$ direction).

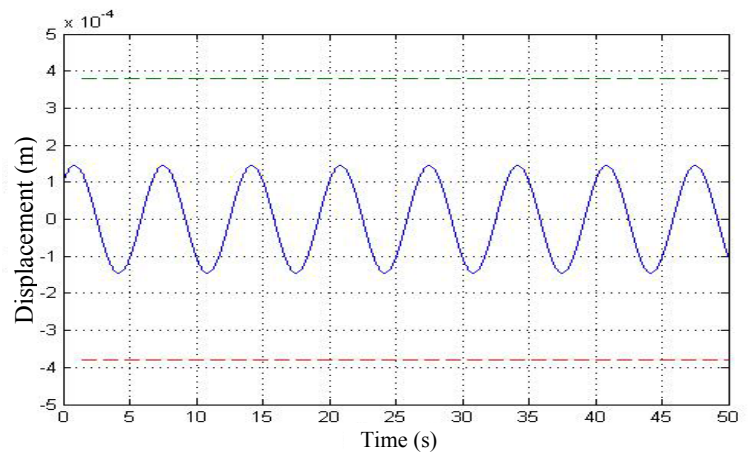

Fig. 13 Gap deviation with flux feedback for four-degrees-of-freedom $(m=100 \mathrm{~kg}, x$ direction).

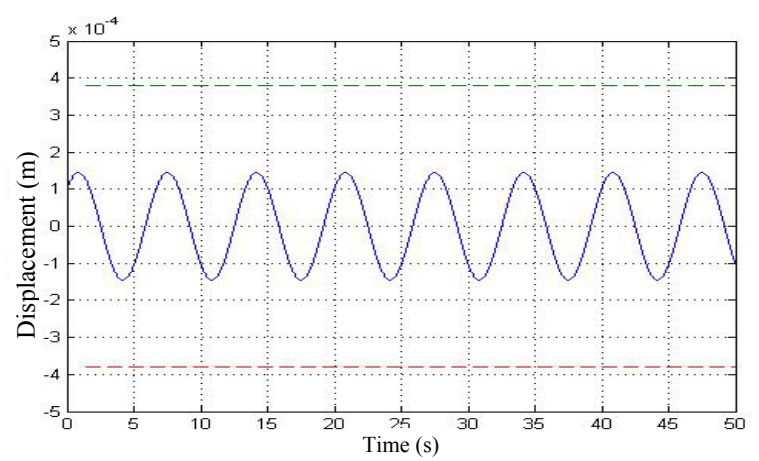

Fig. 14 Gap deviation with flux feedback for four-degrees-of-freedom ( $m=100 \mathrm{~kg}, y$ direction).

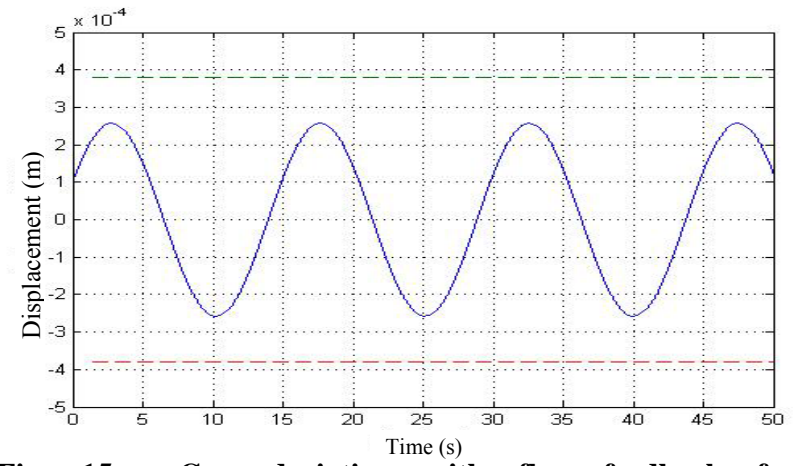

Fig. 15 Gap deviation with flux feedback for four-degrees-of-freedom $(m=500 \mathrm{~kg}, x$ direction). 

Magnetic Bearings Using Flux Feedback

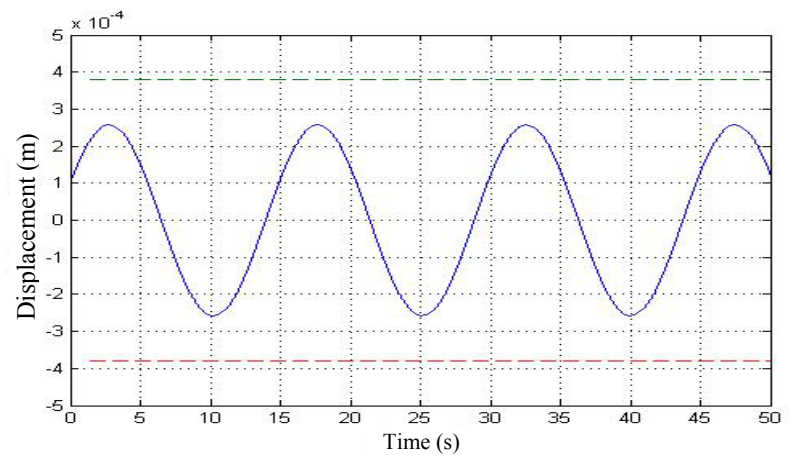

Fig. 16 Gap deviation with flux feedback for four-degrees-of-freedom $(m=500 \mathrm{~kg}, y$ direction).

12-16, the system is well bounded under the effect of the flux feedback loop even if the degree-of-freedom of the system is increased and also the load level is changed $(100 \mathrm{~kg}, 500 \mathrm{~kg})$. This is because of the flux dieback loop that represents linear relationship with respect to the change of the coil current and the air gap displacement which make it possible to decrease in the parameter uncertainties of the $k_{z}, k_{i}$ for the one-degree-of-system and the $k_{s}, k_{i}$ for the four-degrees-of-system.

\section{Conclusions}

In this paper, the open loop characteristics of the electromagnets suspension system which has the flux feedback loop to be applied to the multi-degrees-of-freedom rotational machinery have been dealt with. The flux feedback loop increases the system robustness against the parameter variations even if no active controller is employed. This property comes from the flux feedback loop that is presented in linear combination of the air gap displacement and the coil current which is different from the conventional open loop structure. First, we showed the fundamental mathematical model which has no flux feedback loop, and then introduced the modified mathematical model including the flux feedback loop. Finally, the robustness against the parameter variations for the modified open loop scheme has been achieved by the simulations, even if the load acting on the system has been changed. This modified open loop scheme can be applied to a system which has multi-degrees-of-freedom and frequent load variations such as magnetically levitated systems.

\section{References}

[1] T. Jin, Y. Liu, A novel GNSS weak signal acquisition using wavelet denoising method, in: Proceedings of the 2008 National Technical Meeting of the Institute of Navigation, San Diego, CA, Jan. 28-30, 2008, pp. 28-30.

[2] J.H. Lee, P.E. Allaire, G. Tao, X. Zhang, Integral sliding mode control of a magnetically suspended balance beam: Analysis, simulation and experimentation, IEEE Trans. On Mechatronics 6 (3) (2001) 338-346.

[3] P.K. Sinha, Electromagnetic Suspension: Dynamics and Control, Peter Peregrinus Limited, UK, 1987.

[4] G. Schweitzer, E.H. Eric, Magnetic Bearings: Theory, Design, and Application to Rotating Machinery, Springer, New York, 2009.

[5] T. Hu, Z. Lin, B. Huang, W. Jiang, P. Allaire, Control of balance beam: A linear design approach, in: 8th International Symposium on Magnetic Bearings, Mito, Japan, 2002.

[6] T. Hu, Z. Lin, P. Allaire, Power loss reduction by optimizing current allocation in magnetic bearings, in: 8th International Symposium on Magnetic Bearings, Mito, Japan, 2002.

[7] J. Lindlau, C.R. Knospe, Feedback linearization of an active magnetic bearing with voltage control, IEEE Transactions on Control Systems Technology 10 (1) (2002) 21-31.

[8] Z. Lin, M. Glauser, T. Hu, P.E. Allaire, Magnetically suspended balance beam with disturbances: A test rig for nonlinear output regulation, International Journal of Advanced Mechatronic Systems 1 (1) (2008) 2-9.

[9] G. Li, Z. Lin, P. Allaire, J. Luo, Modeling of a high speed flywheel control test rig with active magnetic bearings, ASME Journal of Vibration and Acoustics 128 (3) (2006) 269-281.

[10] J.H. Lee, P.E. Allaire, W. Jiang, T. Hu, Z. Lin, Cancellation of static and sinusoidal disturbance forces in a magnetic suspension system using exerted force and flux feedback, in: Eighth International Symposium on Magnetic Bearings, Japan, Aug. 26-28, 2002.

[11] J.H. Lee, A study on an effect of the flux feedback on an open-loop characteristic of the magnetic levitation system, in: 13th International Symposium on Magnetic Bearings, Washington, Virginia, USA, Aug. 6-8, 2012. 